\title{
Farmacoterapia geriátrica: o uso de medicamentos e as doenças crônicas não transmissíveis em idosos
}

\author{
Geriatric pharmacotherapy: the use of medications and chronic non-communicable \\ diseases in the elderly
}
Farmacoterapia geriátrica: uso de medicamentos y enfermedades crónicas no transmisibles en ancianos

Ana Paula França Coutinho ${ }^{1 *}$, Rosa Malena Fagundes Xavier ${ }^{1}$, Aníbal de Freitas Santos Júnior ${ }^{1}$, Maria Teresita Fernandez Bendicho' ${ }^{1}$.

\begin{abstract}
RESUMO
Objetivo: Caracterizar a utilização de medicamentos de uso contínuo para tratamento das doenças crônicas não transmissíveis em idosos que participam do programa Universidade Aberta à Terceira Idade. Métodos: Trata-se de um estudo de caráter transversal descritivo, utilizando uma amostragem não probabilística por conveniência. Ocorreu no período de maio a julho de 2019, com coleta de dados mediante a aplicação de um questionário estruturado, contemplando as características sócio demográficas, clínicas e farmacológicas, voltadas para o cuidado em saúde. Resultados: Dentre as Doenças Crônicas Não Transmissíveis mais prevalentes, destacaram-se: hipertensão arterial, diabetes mellitus, hipercolesterolemia e hipotireoidismo. Os fármacos mais utilizados pelos idosos foram losartana potássica, hidroclorotiazida, losartana potássica e hidroclorotiazida associados, cloridrato de metformina, sinvastatina e Atenolol. Conclusão: Pode-se constatar que a idade avançada é um fator que torna essa população susceptível a adquirir doenças crônicas, retirando o indivíduo do estado de homeostase e, portanto, aumentando o uso dos serviços de saúde e a utilização de múltiplos medicamentos para o controle das doenças.
\end{abstract}

Palavras-chave: Doenças não transmissíveis, Idosos, Medicamentos.

\begin{abstract}
Objective: To characterize the use of medications for continuous use to treat chronic non-communicable diseases in the elderly who participate in the Open University program for the Elderly. Methods: This is a cross-sectional, descriptive study, using non-probabilistic sampling for convenience. It took place from May to July 2019, with data collection through the application of a structured questionnaire, covering sociodemographic, clinical and pharmacological characteristics, focused on health care. Results: Among the most prevalent Chronic Non-Communicable Diseases, the following stood out: arterial hypertension, diabetes mellitus, hypercholesterolemia and hypothyroidism. The drugs most used by the elderly were losartan potassium, hydrochlorothiazide, losartan potassium and associated hydrochlorothiazide, metformin hydrochloride, simvastatin and Atenolol. Conclusion: It can be seen that advanced age is a factor that makes this population susceptible to acquiring chronic diseases, removing the individual from the state of homeostasis and, therefore, increasing the use of health services and the use of multiple medications to control disease.
\end{abstract}

Keywords: Noncommunicable diseases, Seniors, Medicines.

\section{RESUMEN}

Objetivo: Caracterizar el uso de medicamentos de uso continuo para el tratamiento de enfermedades crónicas no transmisibles en ancianos que participan en el programa Universidad Abierta para Ancianos. Métodos: Se trata de un estudio descriptivo de corte transversal, utilizando un muestreo no probabilístico por

1 Universidade do Estado da Bahia (UNEB), Salvador - BA. *E-mail: ana_coutinho10@yahoo.com.br 
conveniencia. Se llevó a cabo de mayo a julio de 2019, con recolección de datos mediante la aplicación de un cuestionario estructurado, cubriendo características sociodemográficas, clínicas y farmacológicas, enfocado a la atención de la salud. Resultados: Entre las Enfermedades Crónicas No Transmisibles más prevalentes se destacaron: hipertensión arterial, diabetes mellitus, hipercolesterolemia e hipotiroidismo. Los fármacos más utilizados por los ancianos fueron losartán potásico, hidroclorotiazida, losartán potásico e hidroclorotiazida asociada, hidrocloruro de metformina, simvastatina y atenolol. Conclusión: Se puede observar que la edad avanzada es un factor que hace que esta población sea susceptible a adquirir enfermedades crónicas, sacando al individuo del estado de homeostasis y, por tanto, aumentando el uso de los servicios de salud y el uso de múltiples medicamentos para el control enfermedad.

Palabras clave: Enfermedades no transmisibles, Mayors, Medicamentos.

\section{INTRODUÇÃO}

Durante o envelhecimento pode ocorrer um conjunto de alterações fisiológicas, morfológicas e psicológicas, que modificam o organismo progressivamente, sendo considerado um processo que pode afetar a independência e autonomia dos idosos. As principais doenças relacionadas ao envelhecimento são classificadas como Doenças Crônicas Não Transmissíveis (DCNT) (MACHADO WD, et al., 2017). Para a Organização Mundial da Saúde (OMS) (2013) as DCNT são todas aquelas de progressão lenta e de longa duração. Nesta categoria estão inseridas as doenças cardiovasculares, metabólicas, respiratórias e câncer. As doenças crônicas são auto adquiridas, envolvem múltiplos fatores, podem ser assintomáticas, porém podem ser prevenidas ou ter sua evolução controlada.

Neste sentido, os idosos podem apresentar polimorbidades e geralmente na maioria dos casos necessitam utilizar muitos medicamentos, evento considerado como polifarmácia. Não há consenso na literatura quanto ao conceito de polifarmácia, mas a definição mais adotada na literatura é o "uso de cinco medicamentos ou mais", porém, para fins do Desafio Global de Segurança do Paciente, a OMS adotou a conceituação de "uso rotineiro de quatro ou mais medicamentos simultâneos por um paciente, sejam eles prescritos ou isentos de prescrição", dessa forma, adotamos esse conceito para o trabalho (RAMOS LR, et al., 2016; NASCIMENTO MMG, et al., 2018).

Segundo Alves NMC e Ceballos AGC (2018), com o aumento da idade cronológica, ocorre uma maior prevalência de condições crônicas, predispondo os idosos a necessidade de uma maior utilização de medicamentos, seja para o controle das doenças e/ou para a manutenção da saúde. Assim, a população geriátrica fica mais exposta à polifarmacoterapia e as suas consequências, uma vez que apresentam alterações fisiológicas que modificam a farmacodinâmica e a farmacocinética, contribuindo para sua toxicidade ou a ocorrência de reações adversas (MUNIZ ECS, et al., 2017).

Neste contexto, este estudo teve por objetivo caracterizar a utilização de medicamentos de uso contínuo para tratamento das doenças crônicas não transmissíveis nos idosos que participam do programa Universidade Aberta à Terceira Idade.

\section{MÉTODOS}

Trata-se de um estudo transversal descritivo realizado com idosos que participam das atividades do programa Universidade Aberta à Terceira Idade (UATI) de uma universidade pública localizada no município Salvador, Bahia. A UATI é um programa de extensão universitária que se caracteriza como uma proposta de educação continuada não formal, objetivando a reinserção psicossocial para o pleno exercício da cidadania e desenvolvendo ações educativas de caráter permanente.

O estudo foi realizado com idosos que faziam uso de medicamento contínuo para tratamento prolongado de DCNT. O tamanho da amostra totalizou 75 entrevistados. O processo de amostragem adotada foi a não probabilística por conveniência, que consiste em selecionar aleatoriamente uma amostra da população que seja acessível. Foram incluídos aqueles com idade igual ou superior a 60 anos, de ambos os sexos. Foram excluídos aqueles que não tinham domínio cognitivo para responder ao instrumento de coleta. 
Os dados foram coletados por meio da aplicação de um questionário estruturado, no período de maio a julho de 2019, mediante o consentimento do participante, contemplando as características sócio demográficas, clínicas e farmacológicas, voltadas para o cuidado em saúde. O banco de dados foi confeccionado no programa Excel for Windows ${ }^{\circledR}$ (V. 7.0) e Minitab Statistical Software, com intuito de sistematizar as informações coletadas. O projeto de pesquisa foi submetido à análise do Comitê de Ética em Pesquisa com Seres Humanos, e aprovado pela Plataforma Brasil sob o número de parecer: 3.330 .913 e CAAE: 11465019.7.0000.0057 em 17 de maio de 2019. O termo de Consentimento Livre e Esclarecido foi assinado pelos participantes os quais ficaram de posse de uma cópia, permanecendo outra com o pesquisador. Foi assegurada a confidencialidade dos dados fornecidos para a presente pesquisa.

\section{RESULTADOS}

Foram entrevistados 75 idosos considerando as características sociais, demográficas e econômicas, o que permitiu traçar o perfil do idoso participante do programa de extensão UATI. Observou-se predominância do gênero feminino, com faixa etária entre 60 a 70 anos e cor de pele negra. A escolaridade referida pela maioria foi ensino médio completo, e a situação conjugal mais frequentes foram viúvos(as) e casados(as). Uma maior proporção dos idosos são aposentados, moram com algum familiar, vivem com renda mensal entre um ou mais de um salário-mínimo, obtém seus medicamentos em farmácia privada, e relataram não apresentar dificuldade financeira para adquirir seus medicamentos de uso contínuo (Tabela 1).

Tabela 1 - Características sociais, demográficas e econômicas dos idosos, $\mathrm{n}=75$.

\begin{tabular}{|c|c|c|}
\hline Variáveis & & $\mathbf{N}(\%)$ \\
\hline Sexo & $\begin{array}{l}\text { Feminino } \\
\text { Masculino }\end{array}$ & $\begin{array}{l}71(94) \\
4(5)\end{array}$ \\
\hline \multirow{3}{*}{ Faixa etária (anos) } & $60-70$ & $42(56)$ \\
\hline & $71-80$ & $29(38)$ \\
\hline & $81-90$ & $4(5)$ \\
\hline \multirow{3}{*}{ Raça/Cor } & Negro (a) & $41(54)$ \\
\hline & Pardo (a) & $18(24)$ \\
\hline & Branco (a) & $16(21)$ \\
\hline \multirow{4}{*}{ Situação Conjugal } & Solteiro (a) & $12(16)$ \\
\hline & Casado (a) & $27(36)$ \\
\hline & Viúvo (a) & $28(37)$ \\
\hline & Divorciado (a) & $8(10)$ \\
\hline \multirow{6}{*}{ Escolaridade } & Ensino Fundamental Incompleto & $16(21)$ \\
\hline & Ensino Fundamental Completo & $11(14)$ \\
\hline & Ensino Médio Completo & $29(38)$ \\
\hline & Ensino Médio Incompleto & $4(5)$ \\
\hline & Ensino Superior Completo & $14(18)$ \\
\hline & Ensino Superior Incompleto & $1(1)$ \\
\hline \multirow{4}{*}{ Ocupação } & Aposentado (a) & $49(65)$ \\
\hline & Pensionista & $7(9)$ \\
\hline & Autônomo (a) & 7 (9) \\
\hline & Outros & $12(16)$ \\
\hline \multirow{4}{*}{ Renda Mensal } & < Salário mínimo & $19(25)$ \\
\hline & > Salário mínimo & $25(33)$ \\
\hline & Igual a um salário mínimo & $27(36)$ \\
\hline & Não informado & $4(5)$ \\
\hline \multirow{2}{*}{$\begin{array}{l}\text { Dificuldade financeira em adquirir o } \\
\text { medicamento }\end{array}$} & Sim & $23(30)$ \\
\hline & Não & $52(69)$ \\
\hline \multirow{3}{*}{ Onde adquiri medicamentos } & Farmácia SUS & $23(30)$ \\
\hline & Farmácia Privada & $38(50)$ \\
\hline & Farmácia do SUS e Privada & $14(18)$ \\
\hline \multirow{2}{*}{ Mora } & Com a família & $66(88)$ \\
\hline & Sozinho & $9(12)$ \\
\hline
\end{tabular}

Fonte: Coutinho APF, et al., 2020. 
Quanto à saúde geral da população estudada, os idosos declararam não possuírem plano de saúde, mas procuram o médico numa frequência de a cada seis meses. Ao auto avaliar-se, consideram sua saúde boa. Todos começaram a usar medicamentos a partir de prescrição médica, a maioria utiliza de um a três e os outros fazem uso de quatro ou mais medicamentos de uso rotineiro e concomitante (Tabela 2).

Tabela 2 - Descrição da população estudada segundo a situação geral de saúde.

\begin{tabular}{lll}
\hline Variáveis & N (\%) \\
\hline Plano de saúde & Não & $32(42)$ \\
& A cada seis meses & $43(57)$ \\
\hline \multirow{3}{*}{ Procura o médico com que frequência } & Uma vez no ano & $46(61)$ \\
& Mais de uma vez no ano & $17(22)$ \\
& Só quando doente & $9(12)$ \\
& Muito boa & $3(4)$ \\
\hline \multirow{3}{*}{ Auto avaliação da saúde } & Boa & $10(13)$ \\
& Regular & $34(45)$ \\
& Ruim & $28(37)$ \\
\multirow{2}{*}{ Começou a usar os medicamentos por } & Prescrição médica & $3(4)$ \\
& Indicação de familiar/amigo & $75(100)$ \\
& Conta própria & $0(0)$ \\
\hline \multirow{2}{*}{ Utiliza quantos medicamentos contínuos } & $1-3$ & $0(0)$ \\
& 4 ou mais & $59(78)$ \\
& Fon & $16(21)$ \\
\hline
\end{tabular}

Fonte: Coutinho APF, et al., 2020.

Em relação aos hábitos e estilo de vida, houve uma proximidade na porcentagem da realização e da não realização de alguma dieta alimentar pelas idosas, e o total de idosos faziam dieta. No que se diz respeito a fatores de risco, a maioria delas não consomem bebida alcoólica, não são tabagistas e praticam atividades físicas. Os poucos idosos entrevistados, são ex-etilista, ex-tabagista e não praticam atividades físicas (Tabela 3).

Tabela 3 - Estilo e hábitos de vida dos idosos participantes do projeto de extensão.

\begin{tabular}{|c|c|c|c|}
\hline \multirow[t]{2}{*}{ Variáveis } & & \multicolumn{2}{|c|}{ Sexo } \\
\hline & & Feminino & Masculino \\
\hline \multirow{3}{*}{ Realizam alguma dieta } & \multirow{3}{*}{$\begin{array}{l}\text { Sim } \\
\text { Não }\end{array}$} & $\mathrm{n}(\%)$ & $\mathrm{n}(\%)$ \\
\hline & & $32(42)$ & $4(5)$ \\
\hline & & $39(52)$ & $0(0)$ \\
\hline \multirow{7}{*}{ Fatores de risco } & Etilismo & $20(26)$ & $1(1)$ \\
\hline & Sim & $46(61)$ & $1(1)$ \\
\hline & Não & $5(6)$ & $2(2)$ \\
\hline & Ex- etilista & & \\
\hline & Tabagismo & $1(1)$ & $0(0)$ \\
\hline & $\operatorname{Sim}$ & $61(81)$ & $1(1)$ \\
\hline & $\begin{array}{c}\text { Não } \\
\text { Ex-tabagista }\end{array}$ & $9(12)$ & $3(4)$ \\
\hline \multirow{2}{*}{ Prática de exercícios físicos } & Sim & $52(69)$ & 1(1) \\
\hline & Não & $19(25)$ & $3(4)$ \\
\hline
\end{tabular}

Fonte: Coutinho APF, et al., 2020.

Dentre as DCNT mais prevalentes, destacaram-se: hipertensão arterial e diabetes mellitus, em seguida a hipercolesterolemia e hipotireoidismo (Gráfico 1). O tipo de diabetes, não foi informado pelos idosos. Do total de entrevistados, a maioria tinha histórico familiar dessas comorbidades e tinham HA e DM associados. 
Gráfico 1 - Quantitativo de condições crônicas de saúde dos idosos.

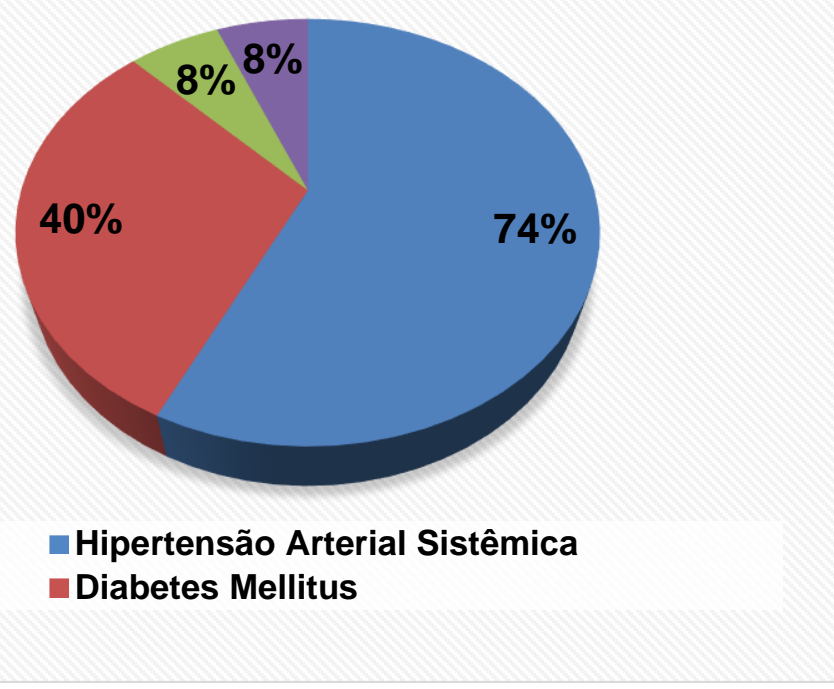

Fonte: Coutinho APF, et al., 2020.

Em relação a farmacoterapia, os medicamentos que os idosos mais utilizavam condiz com as doenças crônicas mais prevalentes nesse estudo, que são: losartana potássica, hidroclorotiazida, losartana potássica e hidroclorotiazida associados, cloridrato de metformina, sinvastatina e atenolol (Gráfico 2).

Gráfico 2 - Percentual dos medicamentos mais utilizados pelos idosos.

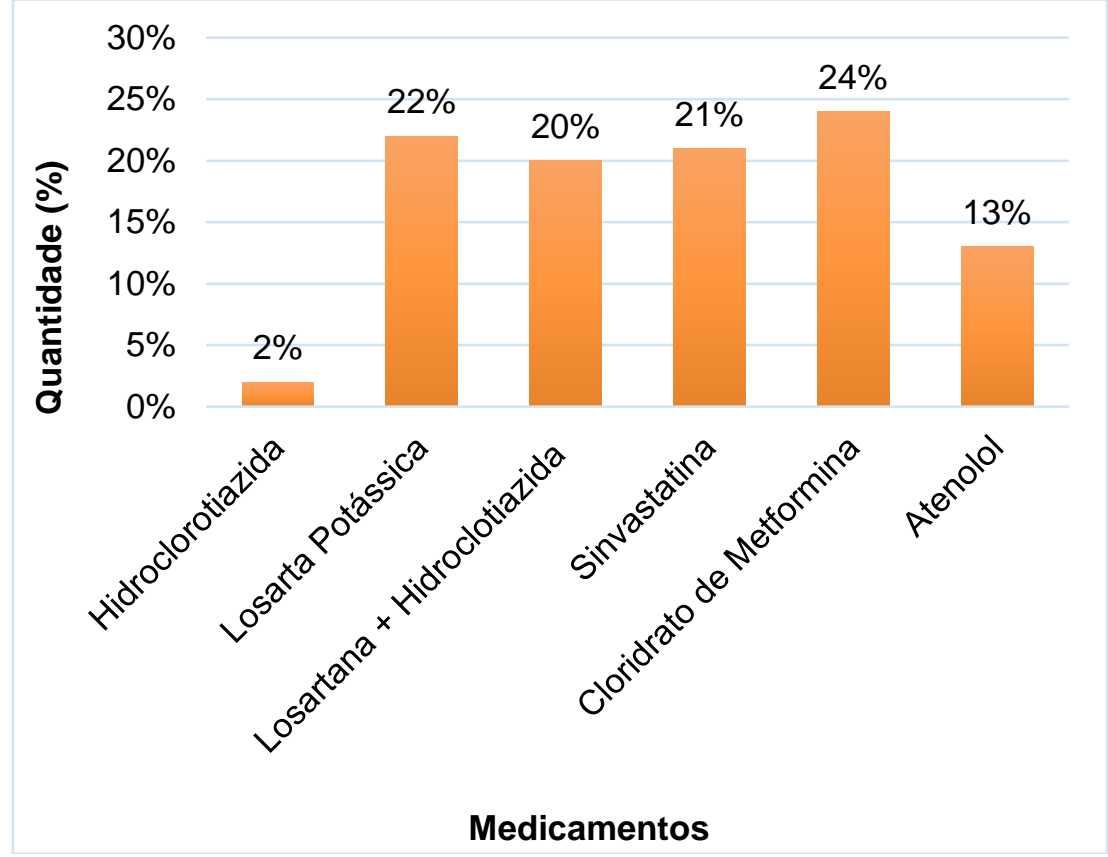

Fonte: Coutinho APF, et al., 2020.

\section{DISCUSSÃO}

A análise dos dados sociodemográficos demonstraram que a maioria dos idosos participantes do programa de extensão eram do sexo feminino, negras, com faixa etária entre 60 e 70 anos, casadas e viúvas. A predominância dessa faixa etária, sendo um maior percentual de mulheres, pode estar associado a uma maior preocupação com o estado de saúde e autocuidado. Além disso, as mulheres são mais propensas a aceitarem a coleta de dados, como o que ocorreu no presente estudo (MUNIZ ECS, et al., 2017). 
Segundo o Instituto Brasileiro de Geografia e Estatística (IBGE) (2019), a expectativa de vida dos homens é de 72,8 anos e das mulheres é de 79,9 anos, o que explica a quantidade de viúvas. Desta forma destacase que a viuvez é uma ocorrência majoritariamente feminina, tendência que se acentua diante de dados demográficos que revelam uma sobrevida maior das mulheres em comparação a longevidade de seus maridos, que por sua vez, são as principais participantes de grupos e atividades voltadas ao segmento idoso (MACHADO WD, et al., 2017; ALVES NMC e CEBALLOS AGC, 2018).

A escolaridade dos idosos deste estudo difere do que se observa na população de idosos em geral, uma vez que a maioria conta com ensino médio ou superior completo, sendo considerado um resultado positivo, tendo em vista que facilita na compreensão e percepção de saúde dos mesmos. A escolaridade é um fator que colabora favoravelmente para o desenvolvimento de todo e qualquer cidadão e é relevante no que concerne aos cuidados com saúde, uma vez que, o baixo nível de escolaridade acarreta dificuldades para população na leitura e interpretação das informações sobre os medicamentos, com risco de uso incorreto e potenciais agravos (LIMA TAM, et al., 2016; MACHADO WD, et al., 2017). O perfil econômico dos idosos entrevistados apresentou uma renda mensal entre um ou mais de um salário-mínimo, em sua maioria advinda da aposentadoria. A renda representa um fator determinante na situação de saúde desse segmento etário, visto que nesta fase da vida existe uma necessidade maior de medicamentos, de serviços de saúde e outros custos que o processo de limitação física acarreta, que chega a comprometer aproximadamente um quarto da renda mensal média de mais da metade da população idosa (SANTOS GS e CUNHA ICKO, 2017; MUNIZ ECS, et al., 2017).

Os idosos relataram sentir uma certa dificuldade em adquirir seus medicamentos de uso contínuo no serviço público de saúde. Considerando o limitado acesso ou não adequado para suprir a população, esse pode ser o motivo da maior parte deles adquirirem seus medicamentos em farmácias privadas. Como a maioria da população estudada morava com algum familiar, o idoso pode se deparar com uma realidade na qual se vê obrigado a amparar familiares desempregados ou doentes, tornando-se o provedor da família (SANTOS GS e CUNHA ICKO, 2017; FERREIRA JÚNIOR E e BATISTA AM, 2018).

O usuário com doenças crônicas utiliza com frequência os serviços das Unidades Básicas de Saúde (UBS), buscando-a por diversas razões: renovação de receitas, consulta de acompanhamento, verificação da pressão e/ou glicemia, entre outras. Segundo Pereira KG et al (2017), quanto maior a utilização de serviços de saúde, maior o uso de medicamentos. A prática da polifarmácia entre idosos suscita especial preocupação, sendo necessário evitar a polifarmácia inapropriada. Entretanto, o uso de múltiplos medicamentos algumas vezes se faz necessário, especialmente quando se tem indicação clara, é efetivo e seguro (MINISTÉRIO DA SAÚDE, 2014; NASCIMENTO MMG, et al., 2018).

A predominância da ausência de tabagismo e do consumo de álcool, da prática de exercício físico, da realização de uma dieta alimentar por uma parcela significativa do público feminino, reafirma que as mulheres se preocupam mais com a saúde do que os homens, que por sua vez em um certo período da sua vida consumiram bebida alcoólica e foram fumantes. O estilo de vida é caracterizado como um conjunto de hábitos, escolhas e costumes vivenciados pelos indivíduos ao longo da vida, e é uma situação marcante no processo saúde-doença desse grupo populacional. Estudos indicam aumento das doenças crônicas em função do crescimento dos quatro principais fatores de risco: dietas não saudáveis, tabagismo, uso nocivo do álcool e inatividade física. Assim, a intervenção nos fatores de riscos, resultaria em redução do número de mortes que ocorre em todo o mundo por DCNT (MACHADO WD, et al., 2017; MALTA DC, et al., 2017; FERREIRA LK, et al., 2018).

Nas doenças crônicas, para se obter um tratamento eficaz também depende fortemente da participação do usuário enquanto sujeito ativo de seu tratamento. Uma atitude de autocuidado que leve a estilos e hábitos mais saudáveis, assim como a adesão ao tratamento, não depende apenas de uma prescrição profissional, mas de uma conscientização do indivíduo sobre sua condição de saúde atual e a relação dela com suas práticas (MINISTÉRIO DA SAÚDE, 2013).

A hipertensão arterial e a diabetes mellitus configuraram-se como as doenças mais informadas pelos entrevistados, que podem estar relacionadas a diversas alterações fisiológicas e bioquímicas geradas pelo 
processo de envelhecimento. Podem provocar sérios graus de incapacidade que afetam tanto os hábitos de vida e o bem-estar do indivíduo, quanto à economia do país. São dois agravos à saúde que merecem especial atenção, em razão de atingirem todas as camadas socioeconômicas, além de se encontrarem entre os mais prevalentes na população (NICOLETTI MA e KUBOTA LT, 2017).

De acordo com a $7^{\circ}$ Diretriz Brasileira de Hipertensão Arterial (2017), HA pode ser definida como uma condição clínica multifatorial caracterizada por elevação sustentada dos níveis pressóricos $\geq 140$ e/ou 90 $\mathrm{mmHg}$. Sua prevalência aumenta progressivamente com o envelhecimento, pois existe uma relação direta $e$ linear da pressão arterial com a idade, sendo a prevalência de HA superior a $60 \%$ na faixa etária acima de 65 anos. A pressão arterial pode ser classificada em normal, pré-hipertensão, estágio 1, estágio 2 e estágio 3 . A medida da pressão arterial é o elemento-chave para o estabelecimento do diagnóstico da HA e a avaliação da eficácia do tratamento (SANTOS SLF, et al., 2016).

O diabetes mellitus (DM) é um grande problema de saúde pública, por se tratar de um distúrbio crônico com elevadas taxas de morbimortalidades, tendo como causa fatores hereditários e ambientais. Caracterizase por hiperglicemia e associadas a complicações, disfunções e insuficiência de vários órgãos. Isto se deve principalmente às complicações crônicas que dela decorrem e que frequentemente têm caráter incapacitante, comprometendo a qualidade de vida dos seus portadores. A classificação do DM baseia-se em sua etiologia, podendo ser do tipo I, tipo II, gestacional e outros tipos específicos (NETTO PPP, et al., 2015; SANTOS SLF, et al., 2016; SOCIEDADE BRASILEIRA DE DIABETES, 2020).

A associação da hipertensão arterial sistêmica (HAS) e diabetes mellitus (DM) é comum, porém aumenta o risco de morte em cerca de sete vezes, principalmente por causas cardiovasculares. O histórico familiar também tem forte influência na pré-disposição de hipertensão arterial sistêmica e diabetes, conforme os resultados desse estudo (RIBEIRO DR, et al., 2020; SOCIEDADE BRASILEIRA DE DIABETES, 2020).

No total da pesquisa foram citados 54 medicamentos de diferentes classes, entre eles os mais citados foram os anti-hipertensivos, antidiabéticos e estatinas. O perfil de medicamentos encontrados, mostrou conformidade com as doenças mais prevalentes na população estudada. $O$ uso de medicamentos pelos idosos em geral é elevado, o que é parcialmente explicado pelo fácil acesso a medicamentos e à baixa frequência de uso de recursos não farmacológicos no manejo de problemas de saúde (PRADO MAMB, et al., 2016).

A primeira abordagem frente ao diagnóstico de hipertensão arterial deve ser com medidas não medicamentosas, conhecidas como mudanças do estilo de vida (MEV), que são eficazes em prevenir a elevação da pressão arterial com a idade e auxiliar no tratamento de pacientes já hipertensos. Para os indivíduos com pressão arterial estágio 1 e risco cardiovascular (CV) baixo e moderado, o tratamento medicamentoso é indicado quando as medidas não farmacológicas não surtirem efeito após um período inicial de pelo menos 90 dias. O tratamento medicamentoso deve ser iniciado de forma imediata quando o indivíduo apresentar pressão arterial estágio 1 e alto risco CV ou DCV estabelecida, ou no caso de hipertensão arterial estágio 2 e 3, independentemente do risco cardiovascular (FUCHS FD, 2016; SOCIEDADE BRASILEIRA DE CARDIOLOGIA, 2016).

Para monoterapia inicial são preferencias os diuréticos tiazídico, tendo como opção a hidroclorotiazida. As tiazidas afetam os mecanismos tubulares renais da reabsorção eletrolítica, aumentando diretamente a excreção de íons sódio e cloreto e privando o corpo do excesso de água. É um fármaco que apresenta mais evidências de efetividade com relação aos desfechos cardiovasculares, com claros benefícios para todos os tipos de eventos (SOCIEDADE BRASILEIRA DE CARDIOLOGIA, 2016; ANVISA, 2020). Pode ser administrada isoladamente, que nesse estudo apresentou uma baixa porcentagem, justificando que possivelmente a maioria dos idosos apresentam RCV alto ou estágios mais avançados, e pode ser administrado em associação com outros fármacos anti-hipertensivos. Há situações em que a indicação de um ou outro grupo ganha destaque, como o caso da losartana potássica - antagonistas de angiotensina II. A losartana liga-se seletivamente ao receptor AT1 e não se liga ou bloqueia outros receptores de hormônios ou canais iônicos importantes na regulação cardiovascular. O seu perfil hemodinâmico em muito se assemelha com o uso dos inibidores da enzima conversora de angiotensina (IECA), sendo o seu atributo principal o de 
melhor tolerabilidade, já que não se associa ao aparecimento de tosse como ocorre com certa frequência com o emprego dos IECA. O uso da Losartana em insuficiência cardíaca congestiva é empregado quando o tratamento com um IECA não é mais considerado adequado (SILVA P, 2010; ANVISA, 2020).

Losartana potássica associado a hidroclorotiazida é indicado quando a terapia combinada for apropriada. Essa combinação apresenta efeito aditivo sobre a redução da pressão arterial, diminuindo a um grau maior do que qualquer um dos componentes isoladamente. Além disso, como resultado de seu efeito diurético, a hidroclorotiazida aumenta a atividade plasmática de renina, a secreção de aldosterona e os níveis de angiotensina II e diminui o potássio sérico (ANVISA, 2020).

O Atenolol (betabloqueador) é considerado escolha inicial em determinadas condições, como a associação de arritmias supraventriculares, enxaqueca, IC e coronariopatia, mas se tratando dessas duas últimas patologias, o atenolol deve ser associado a outros fármacos. Mesmo com porcentagem baixa em relação a prevalência da hipercolesterolemia, o relato do uso da sinvastatina foi expressivo entre os entrevistados. $O$ início da terapia medicamentosa das dislipidemias depende do risco cardiovascular do paciente e do tipo de dislipidemia presente. Na hipercolesterolemia isolada, os medicamentos recomendados são as estatinas, que está indicado em terapias de prevenção primária e secundária como primeira opção (SOCIEDADE BRASILEIRA DE CARDIOLOGIA, 2016 e 2017).

Como não foi especificado nos resultados qual o tipo de diabetes mais prevalente, mas considerando que o diabetes tipo 2 é o que mais acomete os idosos - devido à disfunção da célula beta, com menor produção da insulina e da resistência a esta, também frequente no idoso em função das mudanças corporais que ocorrem com o envelhecimento - então pode ser explicado a prevalência do uso do cloridrato de metformina por esse segmento etário (PRADO MAMB, et al., 2016).

A metformina é um antidiabético oral eficaz, geralmente bem tolerado, frequentemente usado na diabetes tipo 2. Diminui os níveis glicêmicos sem risco de hipoglicemia ou aumento ponderal, tendo a vantagem de ser fácil de administrar e com baixo custo associado (PEIXOTO CILS e RAMALHO C, 2016). Observa-se também que agentes farmacológicos, como a metformina, que apresenta maior segurança a longo prazo, têm diminuído a incidência de diabetes em indivíduos com pré-diabetes (SOCIEDADE BRASILEIRA DE DIABETES, 2020).

O cuidado nutricional também faz parte do tratamento da DM, sendo uma das estratégias mais desafiadoras de mudança do estilo de vida. Bem como o seu papel na prevenção, no gerenciamento da doença e na prevenção do desenvolvimento das complicações decorrentes. As escolhas alimentares promovem efeito direto sobre o equilíbrio energético e, consequentemente, sobre o peso corporal e os níveis pressóricos e de lipídios plasmáticos (SOCIEDADE BRASILEIRA DE DIABETES, 2020).

\section{CONCLUSÃO}

Pode-se concluir que essa população está susceptível a adquirir doenças crônicas, retirando o indivíduo do estado de homeostase e, portanto, aumentando o uso dos serviços de saúde e a utilização de múltiplos medicamentos para o controle das doenças. Os medicamentos que os idosos mais utilizam são Cloridrato de metformina, Losartana potássica e Sinvastatina refletindo a presença de hipertensão arterial, diabetes mellitus e hipercolesterolemia, condições crônicas crescentes no Brasil e no mundo. Tais resultados reforçam a necessidade de ações preventivas para frear o aumento dessas morbidades, diminuindo assim as necessidades medicamentosas, e obtendo melhor qualidade de vida da população ao atingir essa fase da vida.

\section{REFERÊNCIAS}

1. AGÊNCIA NACIONAL DE VIGILÂNCIA SANITÁRIA. Bulário Eletrônico [Brasília]: ANVISA; Disponível em: http://www.anvisa.gov.br/datavisa/fila_bula/index.asp Acesso em: 5 maio 2020.

2. ALVES NMC, CEBALLOS AGC. Polifarmácia em idosos do programa universidade aberta à terceira idade. Journal Of Health \& Biological Sciences. 2018; [s.l.], 6(4):412.

3. FERREIRA JÚNIOR E, BATISTA AM. Atenção farmacêutica a idosos portadores de doenças crônicas no âmbito da atenção primária à saúde. Infarma - Ciências Farmacêuticas, 2018; [s.l.], 30(2):95. 
4. FERREIRA LK, et al. Avaliação do estilo e qualidade de vida em idosos: uma revisão de literatura. Revista Brasileira de Geriatria e Gerontologia, 2018; [s.I.] 21(5):616-627.

5. FUCHS FD. Comparação entre medicamentos para tratamento inicial da hipertensão arterial sistêmica. Uso Racional de Medicamentos: fundamentação em condutas terapêuticas e nos macroprocessos da Assistência Farmacêutica, 2016.

6. INSTITUTO BRASILEIRO DE GEOGRAFIA E ESTATÍSTICA. Expectativa de vida dos brasileiros aumenta para 76,3 anos em 2018. Rio de Janeiro: Instituto Brasileiro de Geografia e Estatística; 2019. Disponível em: https://agenciadenoticias.ibge.gov.br/agencia-noticias/2012-agencia-de-noticias/noticias/26103-expectativa-de-vidados-brasileiros-aumenta-para-76-3-anos-em2018\#: :text=A\%20 expectativa\%20de\%20vida\%20dos,longevidade\%3A\%2079\%2C9\%20anos. Acesso em: 21 abr 2020.

7. LIMA TAM, et al. Acompanhamento farmacoterapêutico em idoso. Arquivo de Ciências da Saúde, 2016; 23(1):52-57.

8. MACHADO WD, et al. Idosos com doenças crônicas não transmissíveis: um estudo em grupos de convivência. Revista Ciência \& Saberes - Facema, 2017; 3(2):444-451.

9. MALTA DC, et al. Doenças crônicas não transmissíveis e a utilização de serviços de saúde: análise da Pesquisa Nacional de Saúde no Brasil. Revista de Saúde Pública, 2017; 51(Supl 1):4s.

10. MINISTÉRIO DA SAÚDE. Diretrizes para o cuidado das pessoas com doenças crônicas nas redes de atenção à saúde e nas linhas de cuidado prioritárias, 2013; 28 p.:il.

11. MINISTÉRIO DA SAÚDE. Estratégias para o cuidado da pessoa com doença crônica. Brasília DF, 2014. (35).162 p.: il.

12. MUNIZ ECS, et al. Análise do uso de medicamentos por idosos usuários de plano de saúde suplementar. Revista Brasileira de Geriatria e Gerontologia, 2017; [s.I.], 20(3):374-386.

13. NASCIMENTO MMG, et al. Polifarmácia: quando muito é demais? Instituto para práticas seguras no uso de medicamentos, 2018; 7(3). Disponível em: https://www.ismp-brasil.org/site/wp-content/uploads/2018/12/BOLETIMISMP-NOVEMBRO.pdf Acesso em: 19 abr 2020.

14. NETTO PPP et al. Ocorrência de complicações crônicas e seus fatores de risco em pacientes diabéticos atendidos pelo programa saúde da família de Muriaé-MG. Infarma - Ciências Farmacêuticas, 2015; 27(2):126.

15. NICOLETTI MA, KUBOTA LT. Benefícios decorrentes de prática do cuidado farmacêutico em hipertensão e diabetes tipo 2 para sua efetivação em unidades de saúde. Infarma - Ciências Farmacêuticas, 2017; [s.l.] 29(4):302.

16. ORGANIZAÇÃO MUNDIAL DE SAÚDE. Escritório Regional para o Sudeste Asiático. (2013). Doenças não transmissíveis: um folheto informativo. Escritório Regional da OMS para o Sudeste Asiático. Disponível em: https://apps.who.int/iris/bitstream/handle/10665/205579/B4980.pdf?sequence=1\&isAllowed=y Acesso em: 19 abr 2020.

17. PEIXOTO CILS, RAMALhO C. A utilização da metformina durante a gravidez. Acta Obstet Ginecol Port, 2016;10(1):43-49.

18. PEREIRA KG, et al. Polifarmácia em idosos: um estudo de base populacional. Revista Brasileira de Epidemiologia, 2017; [s.I.], 20(2):335-344.

19. PRADO MAMB, et al. Diabetes em idosos: uso de medicamentos e risco de interação medicamentosa. Ciência \& Saúde Coletiva, 2016; [s.I.], 21(11):3447-3458.

20. RAMOS LR, et al. Polifarmácia e polimorbidade em idosos no Brasil: um desafio em saúde pública. Revista de Saúde Pública. $2016 \mathrm{Abr} ; 50$ (supl 2):9s.

21. RIBEIRO DR, et al. Prevalência de diabetes mellitus e hipertensão em Idoso. Revista Artigos.Com, 2020.

22. SANTOS GS, CUNHA ICKO. Fatores associados ao consumo de medicamentos entre idosos de uma unidade básica de saúde. Revista Família, Ciclos de Vida e Saúde no Contexto Social, 2017; [s.I.], 5(2):191.

23. SANTOS SLF et al. Educação em saúde para idosos portadores de diabetes e hipertensão: um relato de experiência. Revista de Saúde Pública Santa Catarina, 2016; 9(2):93-104.

24. SILVA P. Farmacologia Cardiovasculorrenal. Farmacologia. Rio de Janeiro: Guanabara Koogan; 2010; 645-646p.

25. SOCIEDADE BRASILEIRA DE CARDIOLOGIA. 7aㅡ Diretriz Brasileira de Hipertensão Arterial. Arq Bras Cardiol, 2016; 107(3Supl.3):1-83.

26. SOCIEDADE BRASILEIRA DE CARDIOLOGIA. Atualização da Diretriz Brasileira de Dislipidemias e Prevenção da Aterosclerose - 2017. Arq Bras Cardiol, 2017; 109(2Supl.1):1-76.

27. SOCIEDADE BRASILEIRA DE DIABETES. Diretrizes da Sociedade Brasileira de Diabetes 2019-2020. 